\title{
Colloidally Induced Smectitic Fines Migration: Existence of Microquakes
}

\author{
K. Krishna Mohan and H. Scott Fogler \\ Dept. of Chemical Engineering, The University of Michigan, Ann Arbor, MI 48109
}

\begin{abstract}
The phenomenon of colloidally induced fines migration is a challenge of both scientific and industrial importance. Its occurrence impacts permeability reduction and alteration of flow pathways in porous media, particulate contaminant migration in groundwater flow, and filtration. The release of smectitic fines is a threshold type of process resulting from discontinuous jumps, called microquakes in the interlayer spacing. There is a critical salt concentration at which these microquakes occur and produce fines migration in the porous media. The changes in the microstructure with decreasing salt concentration were analyzed using $X$-ray diffraction. The transition between crystalline and osmotic swelling regime is also shown to depend on the type of cation. $A$ mathematical model developed using colloidal principles predicts the swelling behavior of smectites in aqueous solutions. The model can predict the transition of swelling from crystalline to osmotic regimes and explain the effect of different cations on the transition.
\end{abstract}

\section{Introduction}

Delineation of the phenomena of colloidally induced fines migration will impact in several areas, such as oil production, landfill remediation, groundwater flow, and soil infiltration. Most oil- and gas-producing formations contain clay minerals that were originally deposited during sedimentation (detrital clay), or precipitated from fluids flowing through the matrix (authigenic clay). Authigenic and detrital clays can cause loss of permeability by several mechanisms. While this loss of permeability, also known as permeability reduction, is undesirable in the area of oil/gas production, it is very beneficial for minimizing leakoff and migration of contaminants in landfill sites. The permeability reduction due to clays is related to colloidal phenomena, which in turn are a function of the ionic composition and $\mathrm{pH}$ of the permeating fluids. The dependence of permeability on the composition of the flowing aqueous fluids is also known as water sensitivity. Prevention of permeability reduction is essential for the successful economic operation of oil and gas wells.

This article describes the colloidally induced mechanisms that cause permeability reduction in porous media containing swelling and nonswelling clays. A number of previous studies have addressed the subject of permeability reduction caused by the water sensitivity of non-swelling clays (Mungan, 1965; Lever and Dawe, 1984; Gray and Rex, 1966; Jones, 1964;

Correspondence concerning this article should be addressed to $\mathrm{H}$. S. Fogler.
Khilar, 1981; Kia et al., 1987; Vaidya, 1991) present in Berea sandstone. Comprehensive research in our laboratory has delineated the roles of $\mathrm{pH}$, ion exchange, and brine concentration on the release or detachment of migrating clays from pore surfaces and subsequent permeability loss of the porous medium. Natural formations contain both swelling and nonswelling clays, and the clay-related permeability loss due to foreign fluids in these formations can be attributed to more than one mechanism. In spite of the abundance of swelling clays in sandstones, relatively few studies have been devoted to the issue of permeability reduction caused by swelling clays (Leone and Scott, 1988; Scheuerman and Bergersen, 1990), and these have used packed columns containing synthetic mixtures of sand and clay (Frenkel et al., 1978; Suarez et al., 1984; Shainberg et al., 1987; Alpertovich et al., 1985; Goldenberg et al., 1984; McNeal et al., 1968; Hardcastle and Mitchell, 1974; Quirk and Schofield, 1955).

Permeability reduction due to clays can be classified as that caused by swelling clays and nonswelling clays. Smectites and mixed-layer clays can swell with changing ionic conditions and eventually disperse and migrate with the flowing fluid. Swelling alone reduces the effective area for flow and causes reduction in permeability. Kaolinites and illites are nonswelling clays that tend to detach from the surface of the pores and migrate when the colloidal conditions are conducive for release. The migrating particles can get trapped in 


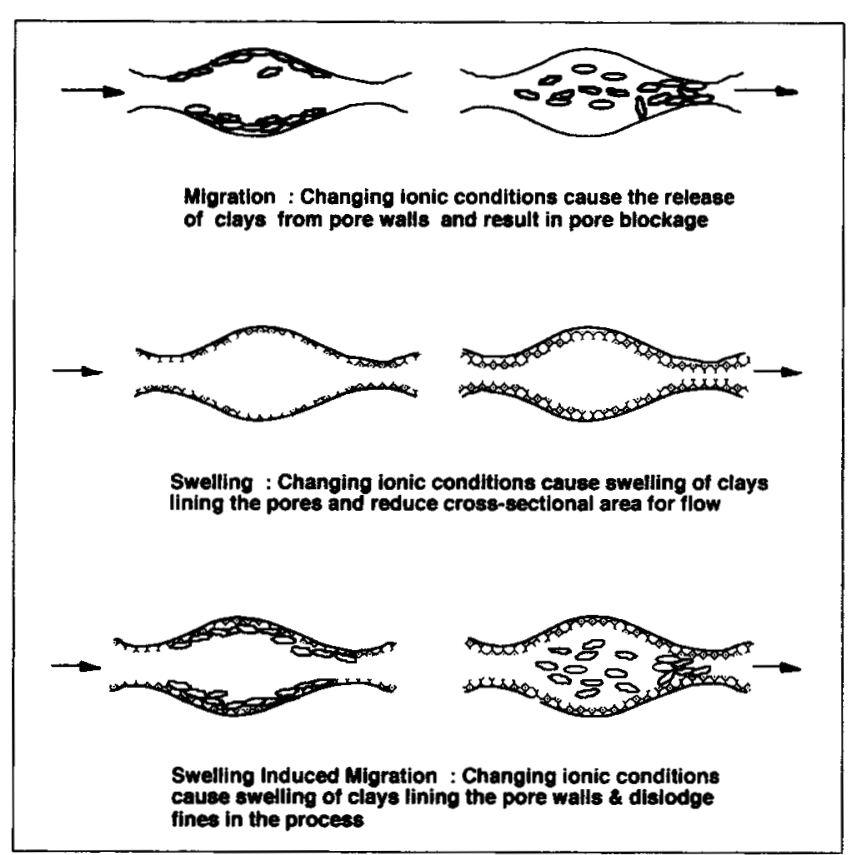

Figure 1. Mechanisms of damage in sandstones containing swelling and nonswelling clays due to changes in aqueous composition.

pore throats, thus causing a reduction in permeability. It has been shown that the clay composition as well as the spatial distribution of clays plays an important role in determining whether permeability reduction problems will be observed in a permeable media (Basan, 1985). Another mechanism of permeability reduction due to swelling clays reported recently is an indirect effect caused by the swelling (Mohan et al., 1993). The depositionary environment could be such that when the clay particles swell, they cause the breakage of fines that are in contact with them. This mechanism is a result of discontinuous jumps, called microquakes, in the swelling process that induce migration. The three possible mechanisms of permeability reduction in sandstones containing swelling and nonswelling clays are shown in Figure 1.

In an earlier article (Mohan et al., 1993), we described a systematic study of permeability reduction due to changes in aqueous composition in sandstones containing swelling and nonswelling clays. In this article, we examine the microstructure of clays using XRD and present a mathematical model for the phenomena of clay swelling, microquakes that lead to fines migration in aqueous solutions.

The results of this study find several applications in the petroleum industry, including design of drilling and completion fluids, selection of flood waters, steam feed waters, and the development of clay stabilizers. The results of this study can be applied to other key areas as well, such as the fate of toxic organics affected by migrating colloids (McDowellBoyer, 1992), the regeneration of filter beds, and erosion of earthen embankments (Khilar et al., 1985).

\section{Materials and Methods}

The experiments in this investigation were conducted on sandstone cores containing swelling and nonswelling clays and on artificial packed beds containing glass beads and clay. The
Table 1. Mineralogical Composition of Sandstones Containing Swelling and Nonswelling Clays

\begin{tabular}{lccc}
\hline & & \multicolumn{2}{c}{ Stevens Sandstone } \\
\cline { 3 - 4 } \multicolumn{1}{c}{ Mineral } & Berea & $7,039 \mathrm{ft}$ & $7,352 \mathrm{ft}$ \\
Sandstone & $(2,145 \mathrm{~m})$ & $(2,241 \mathrm{~m})$ \\
\hline Quartz & 86 & 35 & 32 \\
K-Feldspar & 5 & 18 & 17 \\
Plagioclase & & 34 & 39 \\
Calcite & 1 & 1 & 1 \\
Dolomite & 1 & 1 & \\
Siderite & & 3 & 2 \\
Clays & 7 & 8 & 9 \\
Total & 100 & 100 & 100 \\
CEC (meq/100 g) & $0.5-2.0$ & 2.9 & 4.2 \\
Total Clays (wt. \%) & $5-8$ & 8 & 9 \\
Kaolinte & 90 & 25 & 9 \\
Illite & 9 & 30 & 19 \\
Smectite & Traces & 39 & 29 \\
Mixed layer & Traces & 13 & 28 \\
Chlorite & Traces & 3 & 15 \\
Porosity & $0.19-0.20$ & 0.18 & 0.20 \\
Initial permeability & $>100 \mathrm{md}$ & $<10 \mathrm{md}$ & $<10 \mathrm{md}$ \\
\hline
\end{tabular}

cores were actual field samples supplied by the Department of Energy and Chevron USA from the Stevens Sand formation in the Elk Hills reservoir in California. The mineralogical composition of these sandstones is given in Table 1. The mineralogical composition of Berea sandstone (Churcher et al., 1991), which is an industrial standard, is also given for comparison. The clay content of all three sandstones is similar, while the clay composition of the samples from Stevens Sands is much higher, with significant amounts of expandable clays (mixed layer and smectite). These samples were semiconsolidated and had lower permeability compared to Berea sandstone.

The packed beds were prepared using glass beads (100-150 $\mu \mathrm{m})$ purchased from Polysciences. The clay, Wyoming montmorillonite SWy-2, was obtained from Clay Mineral Repository, University of Missouri, Columbia. All the chemicals used in the experiments were reagent grade.

\section{Preparation of cores and packed beds}

The cores were obtained from Chevron Petroleum Technology Company. The cores were drilled from a slab using liquid nitrogen as the drilling fluid to minimize exposure to aqueous fluids and to preserve the integrity of the semiconsolidated samples, and then flushed with methanol and toluene and saturated with 1-M NaCl. A few cores were saturated with $1-\mathrm{M} \mathrm{KCl}$. The cores were then wrapped in a polyethylene film to prevent moisture loss and stored in a refrigerator until further use.

The packed beds were prepared using acid-washed glass beads, first treated with $0.1-\mathrm{N} \mathrm{HCl}$ for $24 \mathrm{~h}$, and then washed with dilute acid and deionized water. The beads were oven dried and stored until further use. The preparation of clay-sand packed beds involved wetting the glass beads with deionized water and then adding the clay so that the clay was attached to the glass beads. This mixture was then dried at $60^{\circ} \mathrm{C}$ and stored until further use. The packed bed was prepared using a heat-shrinkable Teflon as the enclosure for the beads. Two steel disks (1-in. (25.4-mm) dia.) with several holes drilled in them served as distributors. A distributor and a 


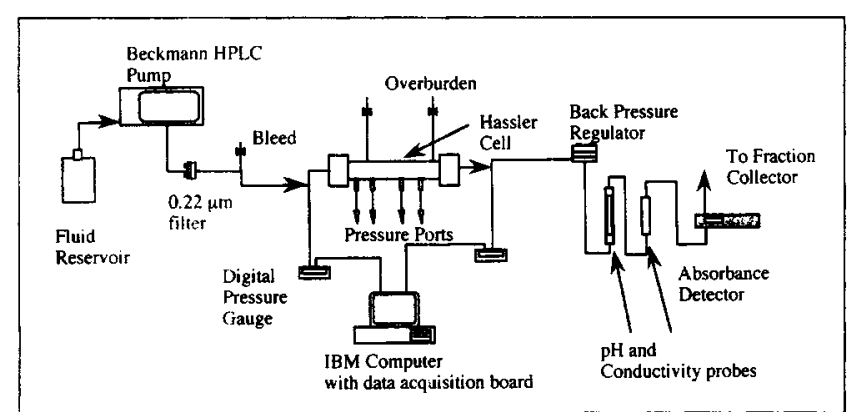

Figure 2. Experimental setup used in coreflood and packed-bed experiments.

steel screen (100 $\mu \mathrm{m}$ nominal diameter) were held together and a heat-shrinkable Teflon was shrunk on one end. The sand-clay mixture was added from the other end and after placing a second screen and distributor on the other side, a Teflon tube was shrunk on to the edges of the distributor. The nominal diameter of the packed beds prepared in this fashion was typically below 1 -in. $(25.4 \mathrm{~mm})$. The heat-shrinking method has the advantage that the tubing flows into the spaces created by the glass beads and eliminates the wall-effects observed in packed beds made using rigid wall material.

\section{Coreflood experiments}

The sandstone cores were vacuum saturated and mounted in a Hassler cell in the setup shown in Figure 2, and pressure was applied around the core to simulate overburden conditions present in the reservoir and to prevent fluid from flowing around the core. The concentrated salt solution was flowed in the forward and reverse directions to ensure that any mobile fines were stabilized. Following this pretreatment, experiments were conducted by changing the salt concentration of the flowing fluid in a stepwise fashion.

The pressure drop across the length of the core was measured using differential digital pressure gauges. The effluent $\mathrm{pH}$ and conductivity were monitored using flowthrough cells and a Cole-Palmer Solution Analyzer. The absorbance of the effluent solution was also monitored using a Gilson flowthrough spectrophotometer with a 560 -nm-wavelength lamp. All the measurements were acquired using a dataacquisition system on an IBM PC.

\section{$X$-ray diffraction measurements}

The mechanisms of the swelling of clays were studied using $\mathrm{X}$-ray diffraction (XRD) with wet samples of clay isolated from the natural sandstone cores as well as pure montmorillonite samples. Three procedures were used for isolating and cleaning the clay samples for XRD.

In one procedure, the powdered core was placed in a separator funnel and treated alternately with methanol and toluene until the toluene solution remained clear for an extended period of time. The material was then treated with methanol and saturated in a brine solution representative of the experiment. The material was next sonicated and the size fraction less than $10 \mu \mathrm{m}$ was separated. An exact separation was not attempted to avoid dispersion of the sample in deion- water. The cleaning method in the second procedure was essentially the same, but the clays were saturated with calcium and then dispersed in deionized water. A centrifugal separation method was used to isolate the $\langle 2-\mu \mathrm{m}$ fraction of the core material. The sample was then saturated with the specified cation. In the third method, the cleaned cores were disaggregated in an ultrasonic bath, and the fine fraction was separated using a coarse separation method and was used for XRD. While all the methods were satisfactory, the third method gave the most consistent results.

The clay sample from the natural sandstone was deposited on a $0.22-\mu \mathrm{m}$ filter paper using a high-speed syringe pump. The clay-coated filter paper was placed on a quartz slide, wrapped with a $0.33-\mathrm{mm}$ Kapton film to prevent moisture loss. In the experiments involving the treatment of samples with multiple solutions, the sample was placed in the filter holder after XRD scanning and fluid flow resumed to equilibrate the clay in the new solution.

XRD measurements on the standard clay were conducted using several methods. In one method, a $0.1 \%$ suspension of clay in a 1-M solution of salt was vacuum filtered onto a filter paper. The filter paper was then mounted in a stainless-steel filter holder and $1 \mathrm{M} \mathrm{NaCl}$ solution was flowed through the sample, followed by the required amount of the desired salt solution, and the XRD was performed as described before. Most of the XRD measurements with the standard clay were performed on a Rigaku Rotaflec rotating anode diffractometer with copper radiation. In another type of measurement, concentrated pastes of clay were made by mixing a salt solution and clay in the ratio 5:1 by weight in plastic vials. After a 24-h equilibration period, the paste was placed in an aluminum holder and covered with Kapton film and the measurements were made on the sample. This method of measuring XRD on concentrated samples is due to Zhou and coworkers (1994).

\section{Results}

\section{Flow experiments}

For single-phase flow in a porous medium, the flow rate through the porous medium and the pressure drop across the length are related by Darcy's law,

$$
Q=K A \frac{\Delta P}{\mu L},
$$

where $A$ is the area of cross section of flow, $\Delta P / L$ is the pressure gradient along the length of the core, and $\mu$ is the viscosity of the flowing fluid. For a constant volumetric flow rate, the effect of changing the aqueous composition of the flowing fluid on the permeability of the porous medium can be examined by the following relation:

$$
\frac{K_{2}}{K_{1}}=\frac{\Delta P_{1}}{\mu_{1}} \frac{\mu_{2}}{\Delta P_{2}}
$$

by accounting for viscosity differences in the flowing fluid.

A typical experiment showing the effect of salt composition on the permeability of a core containing swelling clays is presented in Figure 3. The permeability of the core at any time 


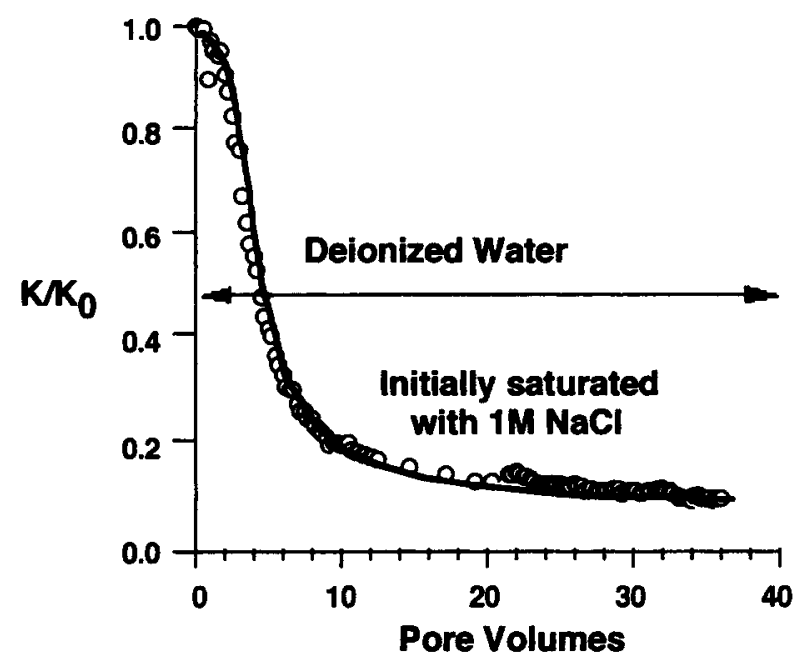

Figure 3. Water shock in Stevens sandstone.

is normalized with respect to the initial permeability of the core with a flowing $1.0-\mathrm{M} \mathrm{NaCl}$ solution and is shown as a function of pore volumes. One observes that changing the salt concentration from $1-\mathrm{M} \mathrm{NaCl}$ solution to deionized water causes a drastic and rapid reduction in permeability. During this reduction, where the permeability of the core reaches approximately $5 \%$ of its original value, clay particles were observed in the effluent from the core. This reduction in permeability is similar to the mechanism reported earlier for Berea sandstone, which contains the nonswelling clays kaolinite and illite (Khilar, 1981; Kia et al., 1987; Vaidya, 1991). The permeability loss is caused by detachment of clay particles lining the pore throats, which migrate and get captured in the pore throats. The Stevens sandstone contains both swelling clays (smectite and mixed layer) and nonswelling clays (kaolinite and illite), and permeability reduction during water shock can be attributed to both these types of clays.

In earlier studies on Berea sandstone (Khilar, 1981; Kia et al., 1987; Vaidya, 1991), it has been reported that the permeability decreases only after the salt concentration falls below a critical salt concentration (CSC). A step reduction in the sodium chloride concentration was carried out on Stevens sandstone, and the results are presented in Figure 4. One observes that the permeability of Stevens sandstone does not decrease as long as the $\mathrm{NaCl}$ concentration remains above $0.5 \mathrm{M}$. The first reduction in permeability occurred when the salinity was changed from 0.5 to $0.25 \mathrm{M}$. Below $0.25 \mathrm{M} \mathrm{NaCl}$ solution, the permeability decreases progressively, as the salt concentration is continually decreased until it approaches that of deionized water.

The critical salt concentrations of other monovalent cations for Stevens and Berea sandstones are shown in Table 2. It is apparent from this table that the minimum salt concentration required to maintain the permeability of Stevens is an order of magnitude higher than that required for Berea. The primary mechanism for initiating permeability reduction in Berea sandstone is the release of kaolinite particles caused by an increase in double-layer repulsion due to reduction in salt concentration. This process is called colloidally induced fines migration. The high, critical salt concentrations at which damage occurs in Stevens compared to Berea sandstone indicate that the onset of permeability reduction cannot be at-

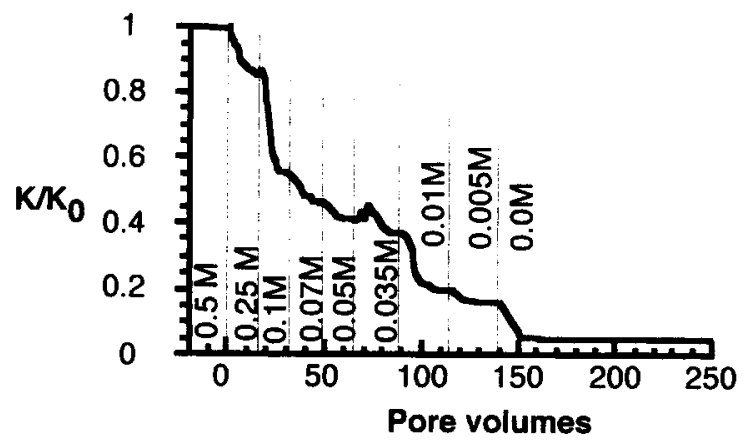

Figure 4. Permeability response of Stevens sandstone to step reduction in $\mathrm{NaCl}$ concentration.

tributed to the kaolinite or illite clays present in the sample. Therefore, the permeability reduction of the Stevens sandstone at the critical salt concentration (ca. $0.25 \mathrm{M}$ ) is attributed primarily to the swelling clays in Stevens sandstones. In order to examine how these clays cause a reduction in permeability due to these changes in salinity, the microstructures of the clays were examined using XRD.

\section{Study of microstructure}

Smectite clay particles are composed of unit sheets known as platelets and are shown in Figure 5. The platelets are essentially two-dimensional structures with a thickness on the order of $10 \AA$, with a lateral dimension on the order of $1 \mu \mathrm{m}$. Each platelet is composed of two tetrahedrally coordinated silica sheets with a octahedrally coordinated alumina sheet between them. The trivalent aluminum in the octahedral sheet is often substituted by divalent ions such as iron and magnesium, while the tetravalent silicon in the tetrahedral sheets can be substituted by trivalent aluminum. These substitutions are termed isomorphic and confer a net negative charge to the platelet. This negative charge is balanced by positive counterions between individual platelets.

The space between individual platelets that dictates the thickness of the clay particle as a whole is filled with counterions and water molecules. The crystalline nature of the platelets and their periodicity enables measurement of the distance between platelets using XRD. Clay material from Stevens sandstone was isolated according to the procedures described earlier and XRD experiments were performed. The clay material was subjected to the same sequence of step decreases in salinity as the coreflood experiments, and an X-ray diffractogram was recorded for each step decrease. The results of this experiment are shown in Figure 6.

The X-ray diffractograms contain several peaks of scattered intensity as a function of the scattering angle. From the

Table 2. Critical Salt Concentrations of Various Salt Solutions in Stevens and Berea

\begin{tabular}{lcc}
\hline & Sandstones & \\
\hline & Stevens & Berea \\
Salt & $\mathrm{M}$ & $\mathrm{M}$ \\
\hline $\mathrm{NaCl}$ & $0.50-0.25$ & 0.07 \\
$\mathrm{KCl}$ & $0.3-0.2$ & 0.03 \\
$\mathrm{CaCl}_{2}$ & $0.3-0.2$ & None \\
\hline
\end{tabular}




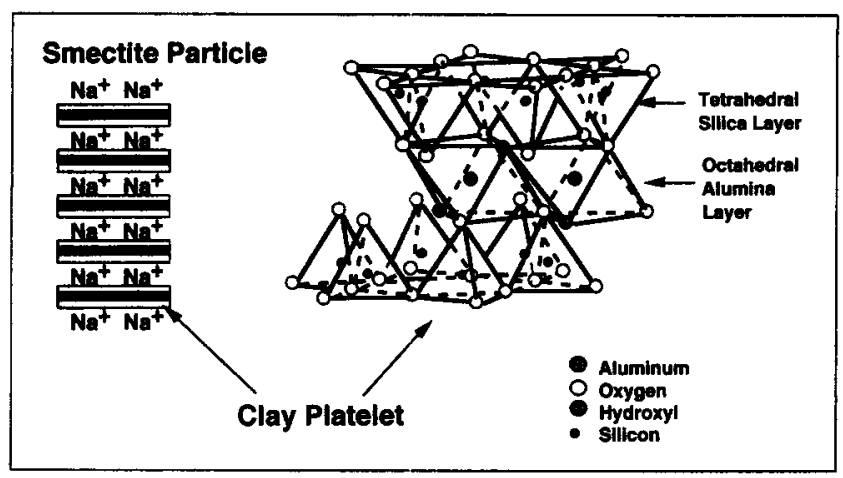

Figure 5. Structure of a swelling clay particle.

locations of the peak positions, the separation distance between identical planes of contiguous platelets, also known as $d$ spacing, can be interpreted using Bragg's law (Moore and Reynolds, 1989). The diffraction pattern of the sample in 1.0 $M$ sodium chloride shows a broad peak at approximately $5^{\circ}$ $(2 \Theta)$ and a small sharp peak adjacent to it. The broad peak shown at $5.35^{\circ}(2 \Theta)$ corresponds to a $d$ spacing of $19 \AA$. This spacing implies that three water layers are retained in between the clay platelets at this ionic strength. The sharp peak corresponds to a $d$ spacing of $15 \AA$, and indicates that there are two water layers between the unit layers of the clay. The other peaks correspond to other minerals and can be used as a reference for orientation of the sample. As the concentration of salt is decreased progressively from $1.0 \mathrm{M}$ to $0.4 \mathrm{M}$ $\mathrm{NaCl}$, there is virtually no change in the intensities and positions of the various peaks. At the next lower concentration, $0.3 \mathrm{M}$, one observes a decrease in the intensity of the $19-\AA$ peak. At a still lower concentration, $0.2 \mathrm{M}$, the area of the $19-\AA$ peak is reduced further and at $0.1 \mathrm{M}$, the peak disappears completely. The base line is higher at roughly $2^{\circ}$; however, there is no visible peak apparent. The changes in the peak areas in this experiment occur at the same concentrations at

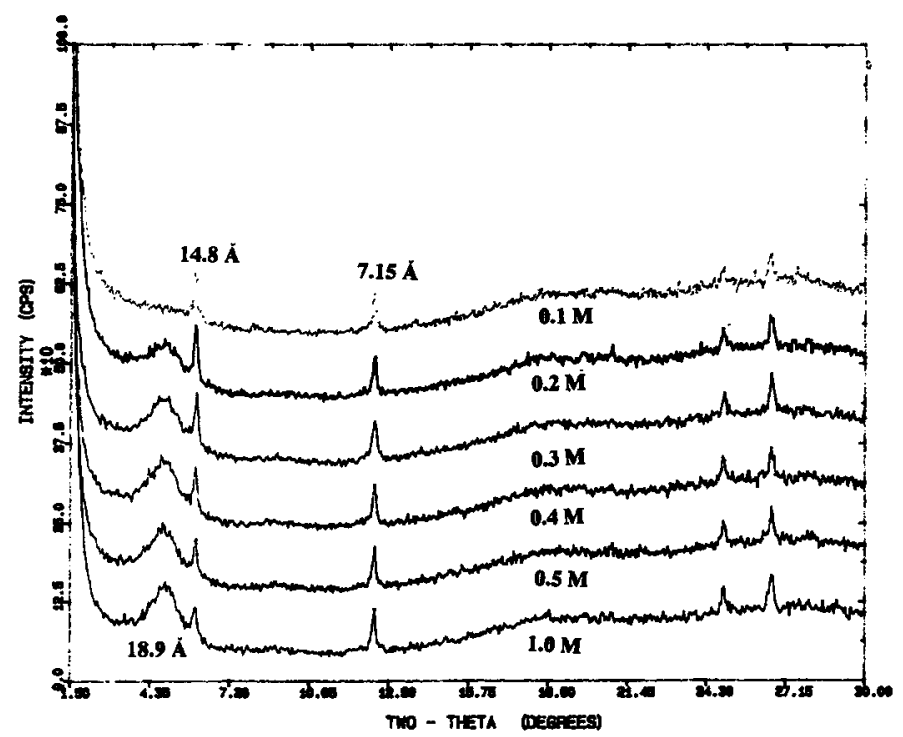

Figure 6. Changes in XRD scans of clay from Stevens due to changes in $\mathrm{NaCl}$ concentration.

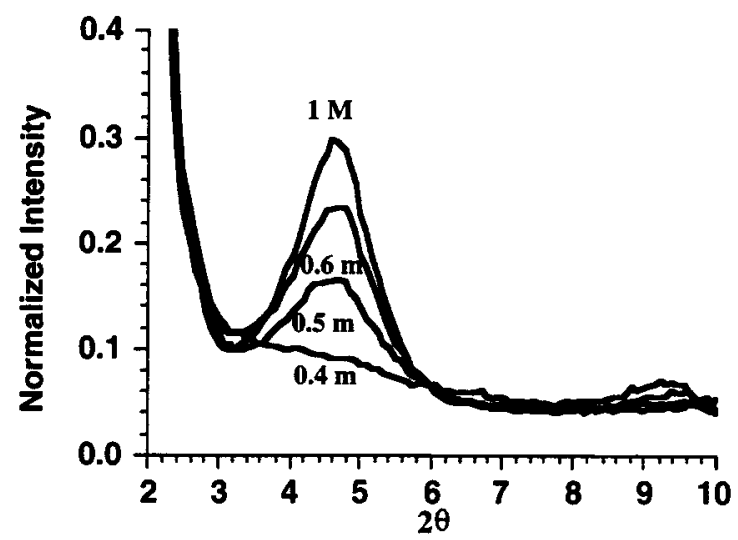

Figure 7. XRD of SWy-2 montmorillonite in $\mathrm{NaCl}$ solutions.

which the drop in permeability is observed in the coreflood experiments. It should be noted that the permeability decrease was larger in the step from $0.25 \mathrm{M}$ to $0.1 \mathrm{M}$, corresponding to the largest decrease in peak area. Consequently, all the permeability changes observed in this concentration range can be related to the movement of the $19-\AA$ peak.

The changes of XRD patterns of the swelling clays from Stevens sandstone in $\mathrm{NaCl}$ solutions are similar to the XRD patterns of a standard clay, Wyoming montmorillonite (SWy-1).The patterns in Figure 7 show that the 19-A peak of the clay is reduced when the sample is treated with solutions of decreasing salt concentrations. The disappearance of the peak area can be the result of two phenomena: (1) a loss of order of periodic arrangement of platelets, and (2) the peak falling outside the detection limit due to the platelet achieving larger dimensions. An increase in platelet separation would make the peaks move to lower angles. The diffracted intensity at the low angles is difficult to detect because of the significant intensity of the primary beam. The progressive decrease in peak area implies that the clay platelets undergo this expansion or loss of order in a gradual fashion, due possibly to the heterogeneous nature of the platelets. As is shown later using a technique with concentrated clay suspensions, the loss of the $19-\AA$ peak is due to the platelets assuming larger separation distances.

The swelling of SWy-1 montmorillonite in various salt solutions has been studied earlier by Norrish (1954). In Norrish's experiments with oriented specimens of clay, the swelling of clays occurs in two regimes, namely crystalline and osmotic. In the crystalline regime, the spacing between clay platelets increases in small discrete increments until the spacing undergoes a large discrete jump (microquake) at a critical concentration. Beyond this concentration, the spacing between the individual platelets is a continuous function of the concentration of the surrounding salt solution. Thus, Norrish hypothesized that the ordering of the platelets in the clay particle is retained, and the loss in intensity of the primary peak at $19 \AA$ is due to this transition to an expanded state. We were able to observe the larger $d$ spacings of this expanded state by using a high solid/liquid ratio technique reported elsewhere (Zhou et al., 1994).

The results of our experiments along with data from the literature are shown in Figure 8. Here, the interplanar spacing is shown as a function of the inverse square root of the 


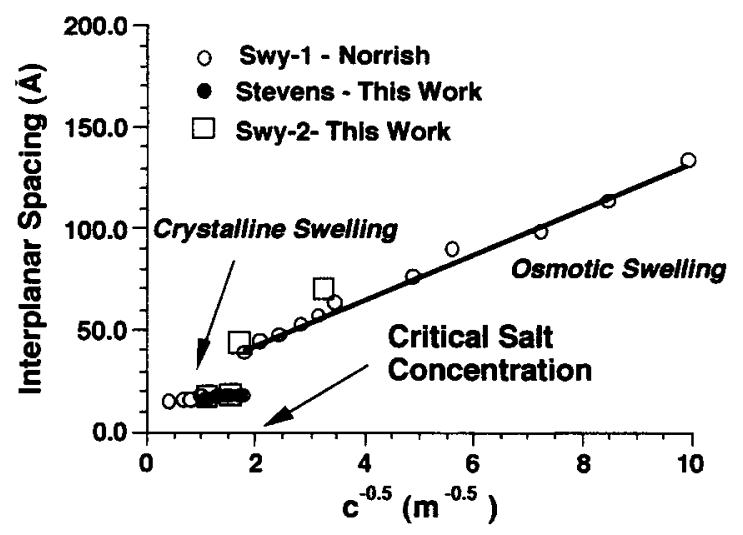

Figure 8. Swelling regimes of montmorillonite in $\mathrm{NaCl}$ solutions.

$\mathrm{NaCl}$ concentration. Both our data and the literature show the crystalline and osmotic swelling regimes of Wyoming montmorillonite (SWy-1 and SWy-2) and smectites from Stevens sandstones in $\mathrm{NaCl}$ solutions.

From the figure we see that the measurements on Stevens sandstones, SWy-2 montmorillonite, and the measurements by Norrish are virtually identical. The two important observations from these data are (1) the discontinuous jump that we refer to as a microquake occurs at the critical salt concentration of $0.25 \mathrm{M}$, and (2) the magnitude of the microquake. This salt concentration of $0.25 \mathrm{M}$ is the concentration at which the permeability reduction is observed in the coreflood experiments.

The coreflood experiments on Stevens and the packed-bed experiments on SWy-1 montmorillonite using sodium, potassium, and lithium chloride solutions reveal that the concentration at which these microquakes are observed is also a function of the type of cation. An XRD analysis of the clays in these solutions shows that the microquake occurs at a higher concentration for lithium-saturated montmorillonite than for sodium-saturated montmorillonite. The potassiumsaturated clay does not expand, even in deionized water. These results are similar to studies by Norrish (1954) on SWy-1 montmorillonite. The $d$ spacings of the clays in various monovalent salt solutions are shown in Figure 9.

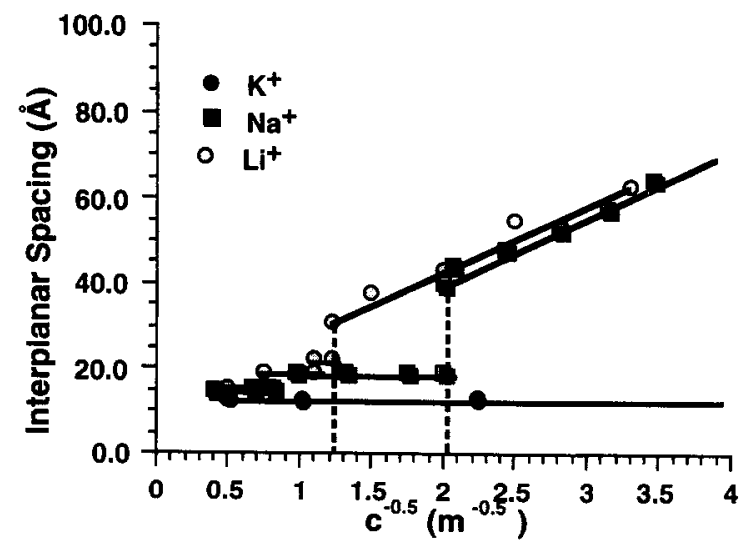

Figure 9. Spacings of Wyoming montmorillonite in various salt solutions.
All of the information in Figures 8 and 9 can be explained by the foliowing predictive model for the critical salt concentration. This model was developed by analyzing the interactions between individual platelets in these various salt solutions using colloidal principles.

\section{Model}

In the previous section, we showed how swelling of clays in various salt solutions can cause loss of permeability in natural sandstones and packed beds. The XRD analysis of clays in equilibrium with various salt solutions corroborates the change in swelling regimes of clays. From a practical viewpoint, this transition in swelling regimes is important in determining the minimum concentration of salt required to prevent permeability reduction. A fundamental understanding of the phenomena governing this transition can help in developing a predictive tool for the critical salt concentration in multicomponent solutions that are more common. In this section, the transition in swelling regimes is examined using a colloidal model.

\section{Background}

The models used to describe crystalline and osmotic swelling and the transition between these regimes are reviewed briefly. A more detailed review of the various models is reported elsewhere (Mohan, 1996). As delineated by Norrish (1954), the swelling of clays can be classified as crystalline and osmotic. In the past, crystalline swelling was analyzed as an outcome of the balance between the repulsive hydration energy of cations and the electrostatic attractive forces between cations and the negatively charged plates (Norrish, 1954; Slade et al., 1991). These two factors can at best only explain limited swelling in highly charged smectites and the dependence of basal spacing on the type of cation in a qualitative manner. However, this analysis cannot account for the sudden transition between crystalline swelling and osmotic swelling. Additional forces that are presumed to affect the crystalline swelling include forces responsible for surface hydration (dispersion and hydrogen bonding), van der Waals, and Born repulsion between clay layers (Low, 1987). The Born repulsion forces are attributed to the overlapping of electron clouds of ions on the surfaces, and can be calculated using empirical potential functions that describe interaction between ions (Israelachvili, 1992).

Traditionally, osmotic swelling has been explained using the classic DLVO theory of particle interaction as a sum of attractive van der Waals forces and repulsive electrostatic forces due to overlap of the electrical double layers of individual particles (Norrish, 1954; Quirk and Schofield, 1955). The electrostatic repulsion force depends on the solution conditions and the nature of interaction between the clay surfaces. This interaction can range from constant charge to constant potential. For constant charge, the charge on the surface remains constant at all distances of separation between individual platelets. In the case of the constant potential, the ions alter the charge of the surface with distance of separation in order to maintain a constant electrostatic potential on the surface of the particles. The magnitude of the electrostatic repulsion is significantly different for these two cases at short separation distances. Several earlier researchers have de- 
scribed the montmorillonite particles as having constant potential surfaces based on electrophoretic mobility data (Horikawa et al., 1988; Delgado et al., 1986; Miller and Low, 1990). As reviewed elsewhere, the interpretation of zeta potentials from mobility data is complicated by surface conductance, double-layer polarization, and the polydispersity of the particle size and charge (Mohan, 1996). Consequently, the assumption of constant potential has to be verified with other experimental measurements.

In addition to van der Waals and electrostatic forces, the swelling of clays may also have contributions from short-range repulsive forces and hydration forces (Low, 1987; Israelachvili, 1992). While the existence of repulsive hydration forces between hydrophilic surfaces is established, the range and magnitude of these forces as well as their dependence on electrolyte concentration for clay particles are not established. The hydration forces are estimated by measuring the total force between particles and subtracting the van der Waals and electrostatic components (Viani et al., 1983; Low, 1987). Consequently, the accuracy of the estimated magnitude and range of hydration forces are strongly dependent on the accuracy of the estimated van der Waals and electrostatic terms. Low and coworkers assume that the electrostatic interaction between clay particles can be described using a constant potential boundary condition based on zeta potentials interpreted using experimental measurements of electrophoretic mobility (Low, 1987). This assumption led them to conclude that the hydration forces dominate the particle interaction to separation distances of $10 \mathrm{~nm}$. However, as mentioned earlier the interpretation of zeta potentials from experimentally determined electrophoretic mobilities is complicated and can lead to erroneous conclusions if all the relevant factors are not taken into account. In view of the uncertainty in the range and magnitude of the hydration forces and other short-range forces. our model assumes that the Born repulsive forces are representative of all non-DLVO forces. As shown in the following subsections, the short-range Born repulsion combined with electrostatic repulsion can satisfactorily explain the total force (swelling pressure) and the observed transition in swelling regimes.

\section{Mathematical model for clay swelling}

The model system used in calculating the interaction energy is shown in Figure 10. Considering the large inplane or $b$ dimension of the platelets compared to their separation distance, the platelets can be assumed to be infinite sheets. The platelets are considered to be uniformly negatively charged, and the value of the negative charge density can be calculated from the exchange capacity and the surface area. We adopt the theory that the counterions are distributed in two layers adjacent to each platelet. Thus each platelet has an associated Stern layer and diffuse layer. The distribution of the ions in these two regions is expected to be dependent on the thermodynamics of ion distribution between the two layers.

The total interaction potential, $V_{T}$, between these two platelets is modeled as a sum of three contributions, namely van der Waals attraction, electrostatic, and Born repulsion, that is,

$$
V_{T}=V_{\mathrm{vdw}}+V_{R}+V_{\mathrm{Born}}
$$

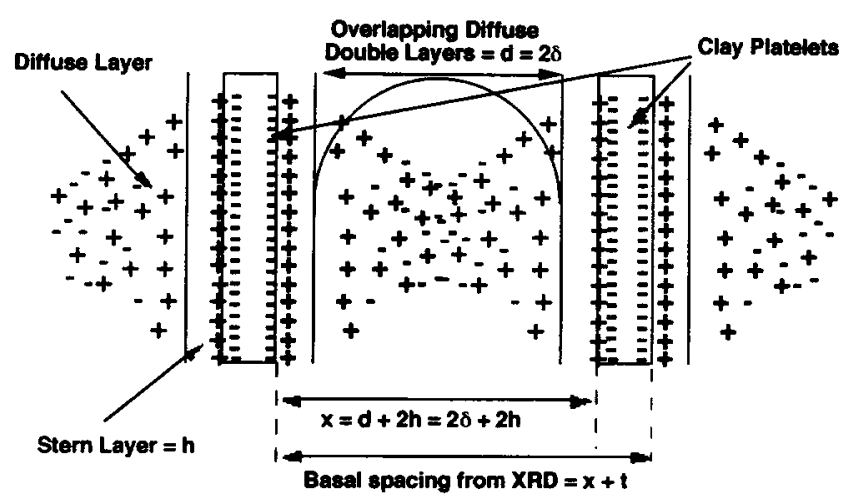

Figure 10. Model system of clay interaction.

van der Waals Attraction. The van der Waals attractive energy between the clay platelets is taken as the interaction of two parallel plates between three films. The van der Waals interaction of semiinfinite plates across three films was formulated by Ninham and Parsegian (1971). The corresponding equation that accounts for the finite thickness of clay platelets is

$$
V_{\mathrm{vdw}}=-\frac{A_{\mathrm{eff}}}{12 \pi}\left[\frac{1}{x^{2}}+\frac{1}{(x+2 t)^{2}}-\frac{2}{(x+t)^{2}}\right]
$$

where

$$
A_{\mathrm{eff}}=\frac{A_{232}(d+2 h)^{2}}{d^{2}}-\frac{A_{123}(d+2 h)^{2}}{(d+h)^{2}}+A_{121}
$$

and

$$
x=d+2 h
$$

where $x$ in Eq. 4 is the distance between the planes of the Stern layer of two adjacent platelets. The distance $d$ is the thickness of the overlapping double layers. The thickness of the platelet $t$ has been measured to be $9.6 \AA$ (van Olphen, 1977). The Stern layer thickness $h$ is taken as $5 \AA$, which is close to the hydrated radius of the sodium ion. The constants $A_{232}, A_{123}, A_{121}$ were calculated from expressions provided by Israelachvili (1992) with values for dielectric constants and refractive indices of the media. The dielectric constant of the Stern layer is taken to be 10 , while values from the literature were used for the other media (Israelachvili, 1992). The calculated values of these constants are shown in Table 3 .

Born Repulsion. The Born repulsion energy between a plane and a sphere has been calculated by Prieve and Ruckenstein (1978) using a 12-power repulsive potential for overlapping of electron clouds at close separations. The same

Table 3. Constants Used in the Calculation of the Total Interaction Energy

\begin{tabular}{lc}
\hline$h$ & $5 \AA$ \\
$t$ & $9.6 \AA$ \\
$\sigma$ & $2.9 \AA$ \\
$A_{123}$ & $-4.92 \times 10^{-15} \mathrm{erg}$ \\
$A_{232}$ & $1.85 \times 10^{-14} \mathrm{erg}$ \\
$A_{131}$ & $1.99 \times 10^{-13} \mathrm{erg}$ \\
\hline
\end{tabular}


power dependency is used to calculate the repulsive energy between two plates of finite thickness. In this case, the Stern layer and the clay platelet were treated as one unit, with properties equal to the water in the Stern layer. The reasoning behind this approach is that the overlapping of the Stern layers causes repulsion due to the interaction of the electron clouds of the bound solvent molecules. The Born constant is taken as $A_{232}$ of the Hamaker constants for interaction across the overlapping double layers. The other constant in this expression is the collision diameter, which is taken as $2.9 \AA$, which is very close to the diameter of the water molecule:

$$
V_{\text {Born }}=\frac{A_{232} \sigma^{6}}{720 \pi}\left[\frac{1}{d^{8}}+\frac{1}{(d+2 t+2 h)^{8}}-\frac{2}{(x+t+h)^{8}}\right] .
$$

Electrostatic Repulsion. The electrostatic interaction is calculated using expressions available in the literature for the case of constant surface potential and constant surface charge (Honig and Mul, 1971). In constant potential simulations, the potential at the Stern plane is fixed, and in the constant charge assumption, the diffuse layer charge, which is equal to the sum of the surface charge and the Stern charge, is fixed. The constant diffuse layer charge assumption implies that as the platelets are brought together, the ions do not transport into the Stern layer, thus keeping the distribution of ions between these layers constant. Figure 11 shows the overlapping double layers with the potential and charge at various locations. The electrostatic repulsion energy is related to the midplane scaled potential $U$, scaled Stern plane potential $Z_{\delta}$ at a platelet separation of $2 \delta$, and $Z_{x}$ at infinite platelet separation by the following expressions involving elliptical integrals of the first and second kind.

The equations for calculating the electrostatic repulsive energy are:

\section{Constant Potential}

$$
\begin{aligned}
& V_{R}=\frac{B}{4}\left[\cosh \left(\frac{Z_{\infty}}{2}\right)-1\right]-\frac{B \kappa \delta}{32}\left(3 e^{U}-2-e^{-U}\right) \\
&-\frac{B}{8} \sqrt{2 \cosh \left(Z_{\infty}\right)-2 \cosh (U)} \\
& E\left(e^{-U}, \frac{\pi}{2}+\frac{B}{4} e^{U / 2}\left\{E\left(e^{-U}, \frac{\pi}{2}\right)-E\left[e^{-U}, \sin ^{-1} e^{\left[\left(U-Z_{\infty}\right) / 2\right]}\right]\right\}\right.
\end{aligned}
$$

$$
\kappa \delta=2 e^{-U / 2}\left\{F\left(e^{-U}, \frac{\pi}{2}\right)-F\left(e^{-U}, \sin ^{-1} e^{\left[\left(U-Z_{x}\right) / 2\right]}\right)\right\}
$$

\section{Constant Charge}

$$
\begin{gathered}
V_{R}=\frac{B}{4}\left[e^{\left(Z_{\curvearrowright} / 2\right)}-1\right]-\frac{B \kappa \delta}{32}\left(3 e^{U}-2-e^{-U}\right) \\
+\frac{B}{8}\left(Z_{\delta}-Z_{\infty}\right) \sinh \left(\frac{Z_{\infty}}{2}\right) \\
+\frac{B}{4} e^{U / 2}\left\{E\left(e^{-U}, \frac{\pi}{2}\right)-E\left[e^{-U}, \sin ^{-1} e^{\left[\left(U-Z_{x} / 2\right]\right.}\right]\right\} \\
\kappa \delta=2 e^{-U / 2}\left\{F\left(e^{-U}, \frac{\pi}{2}\right)-F\left(e^{-U}, \sin ^{-1} e^{\left[\left(U-Z_{\infty} / 2\right]\right)}\right\}\right.
\end{gathered}
$$

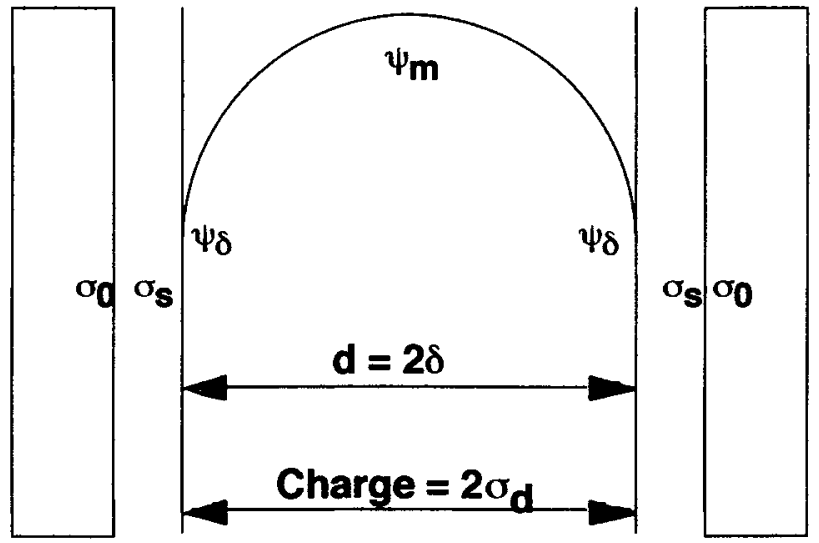

Figure 11. Potential and charge distribution between clay platelets.

$$
\begin{gathered}
2 \cosh \left(Z_{\delta}\right)-2 \cosh (U)=4 \sinh ^{2}\left(\frac{Z_{\infty}}{2}\right) \\
\sigma_{\infty}=\frac{4 n v e}{\kappa} \sinh \left(\frac{Z_{\infty}}{2}\right) \\
\sigma_{0}+\sigma_{s}+\sigma_{d}=0,
\end{gathered}
$$

where

$$
\begin{gathered}
B=64 \frac{n k T}{\kappa}, \quad U=\frac{e \psi_{m}}{k T} \\
Z_{\delta}=\frac{e \psi_{\delta}}{k T} \quad \text { for } \delta \neq \infty, \quad Z_{\propto}=\frac{e \psi_{\delta}}{k T} \quad \text { for } \delta=\infty
\end{gathered}
$$

Constant Potential Simulation. The values of the diffuse layer potential needed for these simulations were obtained from two sources. Under the constant potential assumption, Delgado and coworkers (1986) reported an empirical relationship between the zeta potential and the $\mathrm{NaCl}$ concentration in the concentration range of $10^{-4} \mathrm{M}$ to $0.1 \mathrm{M}$. This relationship is assumed to be valid at higher salt concentrations. The total interaction energy in the constant potential simulation for three different concentrations of NaCL $(0.5$ $\mathrm{M}-0.1 \mathrm{M}$ ) was calculated. These simulations showed that at all of the three concentrations, the total interaction energy exhibits a strong minimum at $12-\AA$ plate separation, which corresponds to a $d$ spacing of $22 \AA$. This value is reasonably close to the experimentally observed value of $19 \AA$ at $0.5 \mathrm{M}$. However, as the concentration is decreased to $0.25 \mathrm{M}$, the minimum remains deep, suggesting that the platelets do not move, contradicting experimental observation. In addition, the constant potential simulation does not predict the experimentally observed swelling pressures in $10^{-4} \mathrm{M} \mathrm{NaCl}$ (Viani et al., 1983). Therefore, it is concluded that interaction between clay platelets does not follow a constant potential.

Constant Charge. The value of the diffuse layer charge is needed to calculate the total interaction energy using the constant diffuse layer charge boundary condition. Using the parameter values shown in Table 3 , the swelling pressure can be calculated for various values of the diffuse layer charge and compared with experimental data from the literature (Viani et al., 1983). These calculations showed that a diffuse layer charge of $2.9 \mu \mathrm{C} / \mathrm{cm}^{2}$ can explain the swelling pressure 


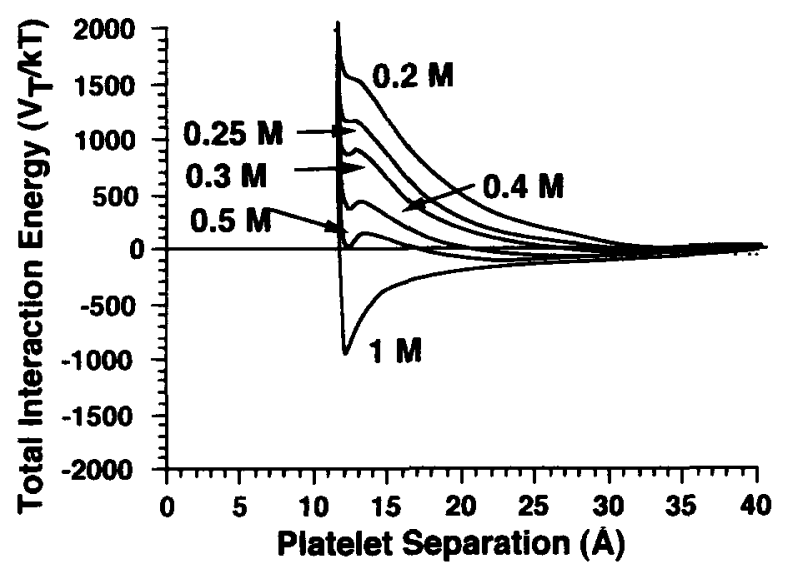

(a)

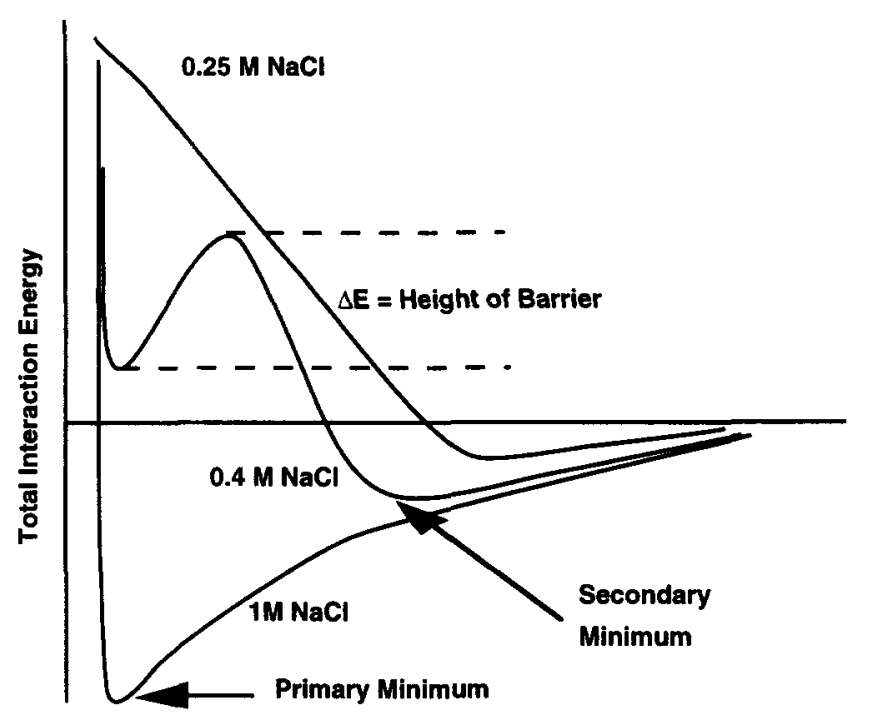

(b)

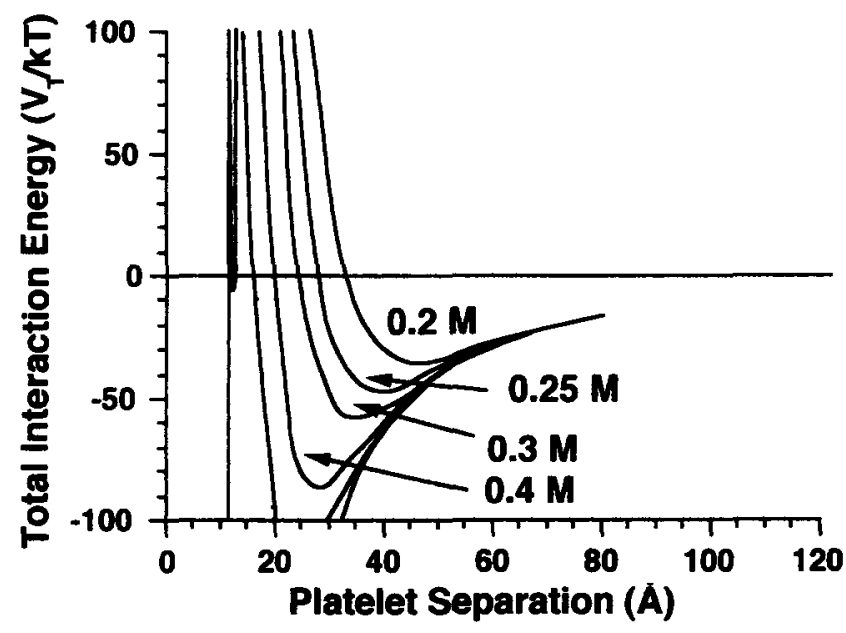

(c)

Figure 12. (a) Variation in primary minimum with salt concentration; (b) transition from primary minimum to secondary minimum; (c) variation in secondary minimum with salt concentration. data of $\mathrm{Na}-\mathrm{SWy}-1$ montmorillonite in $\mathrm{NaCl}$ solutions reasonably well (Mohan, 1996). This value corresponds to balancing approximately $25 \%$ of the total charge that is present on the clay surface as estimated by cation exchange capacity and surface area. The remaining $75 \%$ of the total charge is balanced by the ions in the Stern layer. The swelling pressure data of Viani et al. are available at a concentration of $10^{-4}$ $\mathrm{M} \mathrm{NaCl}$. It is assumed that the diffuse layer charge remains constant, and therefore the charges (i.e., ion) in the diffuse layer do not move into the Stern layer as the salt concentration is increased.

Comparison of Model Predictions and Spacings from XRD. The interaction potential diagrams from our simulations for the constant diffuse layer charge are shown in Figures 12a and 12c. As can be seen from Figure 12a, there is a strong primary minimum in the interaction energy between the clay platelets at a high ionic strength $(1 \mathrm{M})$. As the concentration is decreased, the primary minimum becomes repulsive between 0.5 and $0.4 \mathrm{M}$. This change would cause this spacing to be repulsive (energetically unfavorable), and the particles would try to move away from each other. However, there is a significant energy barrier that would prevent the particles from diffusing away from each other. As the concentration of salt solution is decreased further, the difference between the primary minimum and the energy barrier $(\Delta E)$ decreases, completely disappearing at $0.26 \mathrm{M}$. At this point the platelets continue to move away from each other as long as the force between them is repulsive. However, a point is reached where the forces become attractive again, thus leading to a secondary minimum.

For salt concentrations below $1 \mathrm{M}$, one observes a strong secondary minimum (Figures $12 \mathrm{~b}$ and $12 \mathrm{c}$ ). At a concentration of $0.25 \mathrm{M}$, this minimum occurs at $39 \AA$, which corresponds to a $d$ spacing of $49 \AA$. The platelets that have moved out of the primary minimum can now be located in the secondary minimum, as shown in Figure 12b. The secondary minimum moves to larger separations with decreasing salt concentration, as shown in Figure 12c, and in agreement with the experimental observations. The comparison between the experimental data of Norrish and the prediction is shown in Figure 13 and Table 4. From this figure we can see the transition from crystalline swelling to osmotic swelling regimes, that is, the microquake can be represented as the transition from the primary to the secondary minima in the total interaction energy calculations.

The interaction energy calculated by the constant diffuse

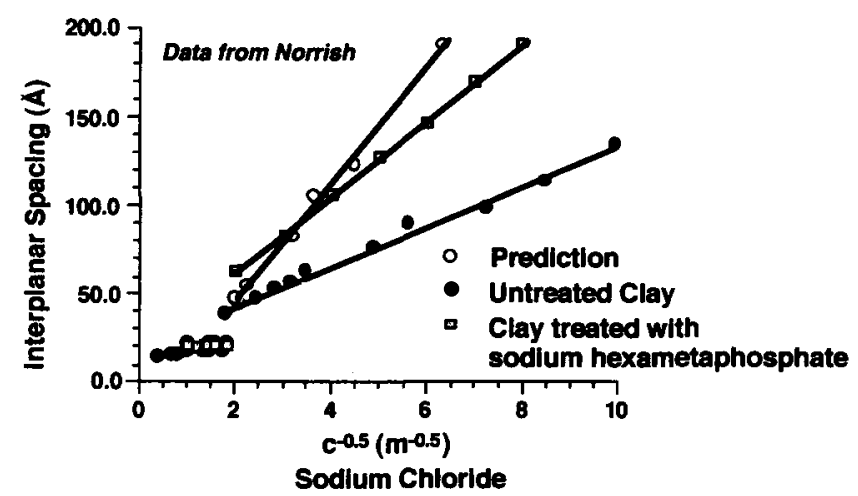

Figure 13. Comparison of theory and experiment. 
Table 4. Comparison between Experiment and Prediction

\begin{tabular}{lccl}
\hline & \multicolumn{2}{c}{ Experiment } & Model \\
\cline { 2 - 3 } & (Untreated Clay) & (Treated Clay) & Pred. \\
\hline CSC & $0.25 \mathrm{M}$ & $0.25 \mathrm{M}$ & $0.26 \mathrm{M}$ \\
Jump Point & $39 \AA$ & $57.8 \AA$ & $49 \AA$ \\
Slope & 11.4 & 21.2 & 34.5 \\
\hline
\end{tabular}

layer charge model and an independent estimate of the charge show that the transition occurs at $0.26 \mathrm{M}$ compared to the experimental value of $0.25 \mathrm{M}$. In addition, the magnitude of the calculated jump ( 22 to $49 \AA$ ) is very similar to the experimentally reported jump of 19 to $39 \AA$. However, the model shows that the spacing in the osmotic regime increases with a larger slope than the experimental observations. One reason for this discrepancy is the interpretation of spacings in the osmotic regime. In the crystalline swelling regime, the spacings can be determined in a straightforward manner using Bragg's law at the observed peak positions. However, in the osmotic regime, the scattered intensity does not produce any peaks and Bragg's law cannot be used to calculate the spacing. These spacings have to interpreted by assuming a geometric model for the clay particle and comparing scattered intensity throughout the low-angle region.

The interpreted spacings are expected to depend on how well the model represents the suspension behavior. The scattering exhibited by the polydisperse suspension of montmorillonite particles of different sizes and charge (Lagaly, 1979) that exists in the form of face-face, edge-edge, and face-edge aggregates is expected to be dependent on the scattering function and the relative number of each of these structures. If the interference from the edge-face and edge-edge structures is destructive, the interpreted spacing using aggregates of disks will be lower than the actual spacing between the clay platelets that compose a particle. This possibility was indeed recognized earlier by Norrish (Norrish and Raussel-Colom, 1963). In a second set of experiments, Norrish showed that the swelling in the osmotic regime exhibited a stronger dependence on the concentration of sodium chloride after pretreating the clays with sodium hexametaphosphate. Phosphate ions are known to adsorb on the edges of clay particles, thus reversing the charge of the edges of the clays (van Olphen, 1977). This treatment will reduce the edge-face flocs, and therefore the scattering curves will be a closer representation of aggregates composed of platelets oriented parallel to each other. As can be observed in Figure 13 and Table 4 , the predicted slope for the osmotic swelling regime is in closer agreement with the experimental data of treated clays than that of the untreated clays.

This model does not account for the retardation of the van der Waals force, which becomes significant for separation distances larger than $5 \mathrm{~nm}$ (Israelachvili, 1992). The retardation effects are not expected to alter the predictions of the transition between primary and secondary minima. The predicted slope in the osmotic regime is expected to be larger with retarded van der Waals forces. Accurate measurements of spacings in this regime would enable quantitative comparisons, with predictions based on retarded van der Waals forces.

This model can also be used to explain the effect of type of cation on the swelling of clays in aqueous solutions. It has

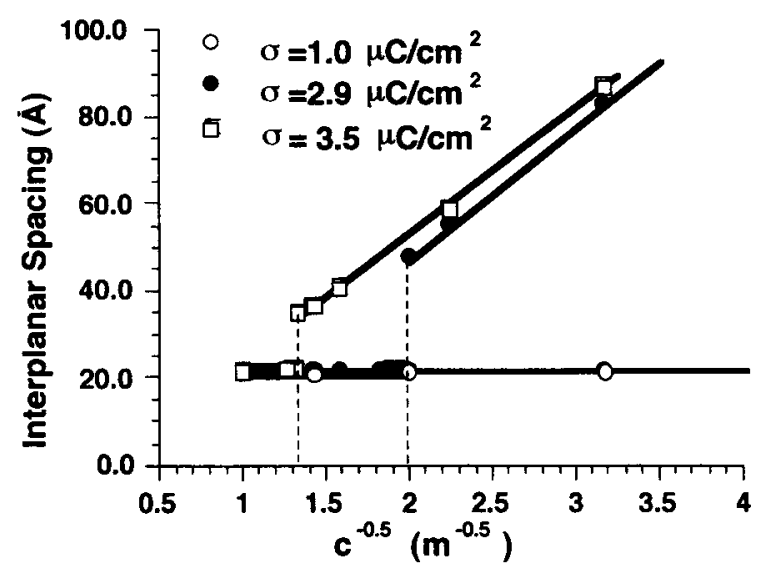

Figure 14. Prediction of $d$ spacings as a function of diffuse-layer charge.

been shown that the potassium-saturated clays do not expand to the osmotic regime, as the concentration of potassium is varied (decreased) (Norrish, 1954). Lithium-saturated clays undergo the transition from crystalline to osmotic swelling regimes at a concentration higher than sodium $(0.66 \mathrm{M})$, as shown in Figure 10. In most mineral systems, the zeta potentials that are an indirect measure of the diffuse layer charge increase in the order of potassium, sodium, and lithium. This behavior suggests that the affinity of potassium to Stern layer is higher than that of sodium, which in turn has a larger affinity than lithium. Thus the diffuse layer charge also increases in this order $(\mathrm{Li}>\mathrm{Na}>\mathrm{K})$. The diffuse layer charge of sodium-saturated SWy-1 montmorillonite was estimated using the swelling pressure data of Viani et al. (1983). However, the same information is not available for potassium- and lithium-saturated clays. We can examine the effect of diffuse layer charge on the concentration at which swelling occurs. These calculations are shown in Figure 14, where it can be seen that at low values of diffuse layer charge, the repulsion is not sufficient to overcome the energy barrier, and therefore the clay will never attain large $d$ spacings. This behavior is very similar to that of the potassium-saturated clays. At high values of diffuse layer charge, the transition occurs at a much higher concentration compared to sodium, which is in agreement with the lithium data where the jump value is lower than in the case of sodium. The model also predicts that the slopes in the osmotic regime are similar for sodium and lithium, which is in agreement with the literature data (Norrish and Rausell-Colom, 1963).

\section{Discussion}

The experimental results just presented show that the changes in the aqueous composition of the fluids can cause permeability reduction in porous media containing swelling clays. The coreflood experiments and microstructure studies clearly identify the solution conditions that result in the transition of clay particles from crystalline to osmotic swelling regimes, which can be thought of as microquakes. These microquakes cause permeability reduction by inducing particulate migration and by the reduction in pore cross-sectional area. As shown elsewhere (Mohan et al. 1993), swelling in the crystalline regime can also cause permeability reduction that can be attributed to particulate migration. In situations where 
the solution concentration is changed but the cation composition is not, the microquakes can be thought of as the primary reason for permeability reduction. Here, when the microquakes occur they dislodge particles on the pore surface that migrate and block the pore throats. A predictive model for the solution conditions that result in these microquakes will be useful for designing fluid compositions for waterflooding and understanding permeability reduction because of changes in the aqueous composition.

The modeling results show that the critical salt concentration at which the transition in the swelling regimes takes place can be described using the DLVO theory and a short-range repulsive interaction. The central point of the theory is that the transition can be modeled as a movement of the platelets from the primary minimum to the secondary minimum at the critical salt concentration. This transition is mediated by increased electrostatic repulsion between the clay surfaces with decreasing salt concentration. The main assumptions in the model are that the short-range repulsive interaction between clay particles can be adequately represented by the Born repulsive term, that the diffuse layer charge is maintained constant during the interaction of the clay particles, and that this charge is constant with ionic strength.

The short-range Born repulsive term serves the purpose of making the primary minimum finite, and combined with the constant diffuse layer charge interaction, can explain the total swelling pressure between the clay platelets at various electrolyte concentrations. It should be pointed out that other forms of short-range repulsion can be used for the same purpose, if good estimates of the range and magnitude of these forces can be obtained.

The assumption of constant diffuse layer charge implies that the ion distribution in the diffuse layer and Stern layer remains constant during a typical collision of the particles. It has been pointed out that during a typical Brownian collision, the exchange of ions between the Stern layer and diffuse layer can be very slow, thus making the diffuse layer charge constant during the collision (Overbeek, 1977). Consequently, the interaction can be treated as a case of constant diffuse layer charge. As shown earlier, the electrostatic interaction based on the constant diffuse layer charge combined with a short-range repulsion can predict both the experimentally measured total force (swelling pressure) at various separations as well as the observed force in transition in swelling regimes.

The third assumption in the model just shown is that the diffuse layer charge is invariant with ionic strength. This assumption was verified with recent measurements of swelling pressures of montmorillonite at electrolyte concentrations ranging from $10^{-4} \mathrm{M}$ to $10^{-2} \mathrm{M}$ (Zhang et al., 1995).

The preceding model provides a method for estimating the critical salt concentration at which swelling proceeds from crystalline to osmotic regimes and leads to substantial permeability reduction in porous media containing swelling clays. The model requires estimates on the diffuse layer charge of the particles at various ionic strengths. It can be extended to multicomponent solutions to predict the critical total ionic strength of the solutions needed at various compositions to prevent permeability reduction. This prediction would require information on the distribution of the various ions between the Stern and diffuse layers at different concentra- tions. This information can be obtained by modeling the electrochemistry of the clay minerals using surface complex models and better measurements of the Stern-layer potential and surface charge.

\section{Summary}

There exists a critical salt concentration (CSC) of sodium chloride at which the permeability of smectitic sandstone decreases significantly. This CSC occurred at $0.25 \mathrm{M} \mathrm{NaCl}$, which is an order of magnitude greater than that for nonsmectitic sandstones such as Berea. In order to elucidate this CSC, XRD studies were undertaken. It was found that the swelling clay particles undergo a transition from the crystalline regime to the osmotic regime at $0.25-\mathrm{M} \mathrm{NaCl}$. At this concentration, the interlayer separation increases by a discrete jump ( $>20 \AA$ ) that can be visualized as a microquake. The microquake is responsible for disrupting the particles on the surface causing them to be dislodged and captured in the pore throats.

A mathematical model using colloidal principles has been developed for predicting the swelling behavior of smectites in aqueous solutions. The model can predict the concentration of $\mathrm{NaCl}$ at which the microquake occurs $(0.25 \mathrm{M})$ and a jump similar in magnitude to the experimentally observed transition. Furthermore, this model can explain the effect of different cations on the transition from crystalline to osmotic swelling regimes. The success of the model in explaining the swelling phenomena in various salt solutions indicates that the clay surfaces are better described as surfaces with constant diffuse layer charge. The model presented here is for single-salt solutions, but can be extended to predict the degree of swelling in multicomponent solutions.

\section{Notation}

$A_{232}=$ Hamaker constant for two surfaces of " 2 "interacting across a medium of 1 , erg

$A_{123}=$ Hamaker constant for surfaces of " 1 " and " 3 " interacting across a medium of 2 , erg

$A_{121}=$ Hamaker constant for two surfaces of " 1 "interacting across a medium of 2 , erg

$A_{\text {eff }}=$ effective Hamaker constant as a function of separation distance, erg

$e=$ electron charge, $\mathrm{C}$

$E(k, \phi)=$ elliptical integral of the second kind

$F(k, \phi)=$ elliptical integral of the first kind

$k=$ Boltzmann's constant

$K=$ permeability of core, $\mathrm{mD}$

$n=$ concentration of electrolyte in solution (particles $/ \mathrm{m}^{3}$ )

$Q=$ volumetric flow rate through core, $\mathrm{cm}^{3} / \mathrm{s}$

$T=$ temperature, $\mathrm{K}$

$t_{\mathrm{Brown}}=$ time scale for a Brownian collision, $\mathrm{s}$

$t_{D I}=$ time scale of charge adjustment in the diffuse double layer, $\mathrm{s}$

$t_{\text {stern }}=$ time scale of charge transfer between diffuse double layer and Stern layer, $\mathbf{s}$

$v=$ valency of electrolyte

\section{Greek letters}

$\delta=$ half-separation distance in the diffuse double layer region, $\AA$

$\epsilon=$ dielectric constant of the bulk solution, $\mathrm{C} / \mathrm{V} / \mathrm{m}$

$\kappa=$ debye Huckel parameter, $\mathrm{m}^{-1}$

$\sigma=$ collision diameter in Born repulsion, $\AA$

$\sigma_{o}=$ charge density of clay platelet, $\mu \mathrm{C} / \mathrm{cm}^{2}$

$\sigma_{d}=$ charge density in the double layer, $\mu \mathrm{C} / \mathrm{cm}^{2}$ 
$\sigma_{s}=$ charge density in the Stern layer, $\mu \mathrm{C} / \mathrm{cm}^{2}$

$\psi_{m}=$ midplane potential, $\mathrm{mV}$

$\psi_{\delta}=$ diffuse layer potential, $\mathrm{mV}$

\section{Literature Cited}

Alpertovich, N., I. Shainberg, R. Keren, and M. J. Singer, "Effect of Clay Mineralogy and Aluminum and Iron Oxides on the Hydraulic Conductivity of Clay-Sand Mixtures," Clays Clay Miner., 33, 443 (1985).

Basan, P. B., "Formation Damage Index Number: A Model for the Evaluation of Fluid Sensitivity of Shaly Sandstone," SPE Tech. Conf. Exhibition, Dallas, TX (1985).

Basu, S., and M. M. Sharma, "Effect of Dielectric Saturation on Disjoining Pressure in Thin Films of Aqueous Electrolytes," J. Colloid Interf. Sci., 165, 355 (1994).

Churcher, R. L., P. R. French, J. C. Shaw, and L. L. Schramm, "Rock Properties of Berea Sandstone, Baker Dolomite, and Indiana Limestone," SPE Int. Conf. Oilfield Chemistry, Anaheim, CA (1991).

Delgado, A., F. Gonzalez-Caballero, and J. M. Bruque, "On the Zeta Potential and Surface Charge Density of Montmorillonite," J. Colloid Interf. Sci., 113, 203 (1986).

Evans, D. F., and H. Wennerstrom, The Colloidal Domain, Where Physics, Chemistry, Biology and Technology Meet, VCH Publishers (1994).

Frenkel, H., J. O. Goertzen, and J. D. Rhoades, "Effects of Clay Content, Exchangeable Sodium Percentage, and Electrolyte Concentration on Clay Dispersion and Soil Hydraulic Conductivity," Soil Sci. Soc. Amer. J., 42, 32 (1978).

Goldenberg, L. C., M. Magaritz, A. J. Amiel, and S. Mandel, "Changes in Hydraulic Conductivity of Laboratory Sand-Clay Mixtures Caused by a Seawater-Freshwater Interface," J. Hydrol., 70, 329 (1984).

Gray, D. H., and R. W. Rex, "Formation Damage in Sandstones Caused by Clay Dispersion and Migration," Clays Clay Miner., 14, 355 (1966)

Hardcastle, J. H., and J. K. Mitchell, "Electrolyte Concentration Permeability Relationships in Sodium Illite-Silt Mixtures," Clays Clay Miner, 22, 143 (1974).

Honig, E. P., and P. M. Mul, "Tables and Equations of the Diffuse Double Layer Repulsion at Constant Potential and at Constant Charge," J. Colloid Interf. Sci., 36, 258 (1971).

Horikawa, Y., R. S. Murray, and J. P. Quirk, "The Effects of Electrolyte Concentration on the Zeta Potentials of Homoionic Montmorillonite and Illite," Colloids Surf., 32, 181 (1988).

Hunter, R. J., Foundations in Colloids Science, Clarendon Press, Ox ford (1989).

Israelachvili, J., Intermolecular and Surface Forces, 2nd ed., Chap. 7, Academic Press, New York (1992).

Israelachvili, J., "Forces Between Surfaces in Liquids," Adv. Colloid Interf. Sci., 16, 31 (1982).

Jones, F. O., "Influence of Chemical Composition of Water on Clay Blocking of Permeability," J. Pet. Technol., 16, 441 (1964).

Khilar, K. C., H. S. Fogler, and D. H. Gray, "Model for Piping-Plugging in Earthen Structures," J. Geotech. Eng. Div. ASCE, 111, 833 (1985).

Khilar, K. C., "Water Sensitivity of Berea Sandstone," PhD Diss., Dept. of Chemical Engineering, Univ. of Michigan, Ann Arbor (1981).

Kia, S. F., H. S. Fogler, M. G. Reed, and R. N. Vaidya, "Effect of Salt Composition on Clay Release in Berea Sandstones," SPE Prod. Eng., 2, 277 (1987).

Lagaly, G., "The Layer Charge of Regular Interstratified 2:1 Clay Minerals," Clays Clay Miner., 27, 1 (1979).

Leone, J. A., and M. E. Scott, "Characterization and Control of Formation Damage During Waterflooding of a High-Clay-Content Reservoir," SPE Reservoir Eng., 3, 1279 (1988).

Lever, A., and R. A. Dawe, "Water Sensitivity and Migration of Fines in the Hopeman Sandstone," J. Pet. Geol., 7, 97 (1984).

Levine, S., and D. G. Hall, "Electric Double Layer Interaction of Highly Charged Platelike Colloidal Particles," Langmuir, 8, 1090 (1992).

Low, P. F., "Structural Component of the Swelling Pressure of Clays," Langmuir, 3, 18 (1987).
McDowell-Boyer, L. M., "Chemical Mobilization of Micron-Sized Particles in Saturated Porous Media Under Steady Flow Conditions," Environ. Sci. Technol., 26, 586 (1992).

McNeal, B. L., D. A. Layfield, and J. D. Rhoades, "Factors Influencing Hydraulic Conductivity of Soils in the Presence of Mixed-Salt Solutions," Soil Sci. Soc. Amer. J., 32, 187 (1968).

Miller, S. E., and P. F. Low, "Characterization of the Electrical Double Layer of Montmorillonite," Langmuir, 6, 572 (1990).

Mohan, K. K., R. N. Vaidya, M. G. Reed, and H. S. Fogler, "Water Sensitivity of Sandstones Containing Swelling and Non-Swelling Clays," Colloids Surf. A; Physicochem. Eng. Aspects, 73, 237 (1993).

Mohan, K. K., "Water Sensitivity of Porous Media Containing Swelling Clays," PhD Diss., Dept. of Chemical Engineering, Univ. of Michigan, Ann Arbor (1996).

Moore, D. M., and R. C. Reynolds, Jr., X-Ray Diffraction and the Identification and Analysis of Clay Minerals, Oxford University Press, Oxford (1989).

Mungan, N., "Permeability Reduction Through Changes in $\mathrm{pH}$ and Salinity," J. Pet. Technol., 17, 1449 (1965).

Ninham, B. W., and V. A Parsegian, "van der Waals Forces across Triple-layer Films," J. Chem. Phys., 52, 4578 (1971).

Norrish, K., "The Swelling of Montmorillonite," Faraday Soc. Discuss., 18, 120 (1954).

Norrish, K., and J. A. Rausell-Colom, "Low-angle X-ray Diffraction Studies of the Swelling of Montmorillonite and Vermiculite," Clays Clay Miner., Proceedings of 10th Conference, Pergamon Press, New York, p. 123 (1963)

Overbeek, J. Th. G., "On the Interaction of Highly Charged Plates in an Electrolyte: A Correction," Mol. Phys., 80, 685 (1993),

Overbeek, J. Th. G., "Recent Developments in the Understanding of Colloid Stability," J. Colloid Interf. Sci., 58, 408 (1977).

Pashley, R. M., and J. P. Quirk, "Ion Exchange and Interparticle Forces Between Clay Surfaces," Soil Sci. Soc. Amer. J., 53, 1660 (1989).

Prieve, D. C., and E. Ruckenstein, "Adsorption and Desorption of Particles and Their Chromatographic Separation," AIChE J., 22, 276 (1976).

Quirk, J. P., and R. K. Schofield, "The Effect of Electrolyte Concentration on Soil Permeability," J. Soil Sci., 6, 163 (1955).

Shainberg, I., R. Keren, N. Alpertovich, and D. Goldstein, "Effect of Exchangeable Potassium on the Hydraulic Conductivity of Smectite-Sand Mixtures," Clays Clay Miner., 35, 305 (1987).

Scheuerman, R. F., and B. M. Bergersen, "Injection-Water Salinity, Formation Pretreatment, and Well Operations Fluid-Selection Guidelines," J. Pet. Technol., 836 (1990).

Schiby, D., and E. Ruckenstein, "The Role of the Polarization Layers in Hydration Forces," Chem. Phys. Lett., 95, 435 (1983).

Slade, P. G., J. P. Quirk, and K. Norrish, "Crystalline Swelling of Smectite Samples in Concentrated $\mathrm{NaCl}$ Solutions in Relation to Layer Charge," Clays Clay Miner., 39, 234 (1991).

Smalley, M. V., "Electrical Theory of Clay Swelling," Langmuir, 9, 2884 (1994).

Spitzer, J. J., "Electrostatic Calculations on Swelling Pressure of Clay-Water Dispersions," Langmuir, 5, 199 (1989).

Suarez, D. L., J. D. Rhoades, R. Lavado, and C. M. Grieve, "Effect of pH on Saturated Hydraulic Conductivity and Soil Dispersion," Soil Sci. Soc. Amer. J., 48, 50 (1984).

Vaidya, R. N., "Fines Migration and Formation Damage," PhD Diss., Dept. of Chemical Engineering, Univ. of Michigan, Ann Arbor, (1991).

van Olphen, Introduction to Clay Colloid Chemistry, Oxford Univ. Press, Oxford (1977).

Viani, B. E., P. F. Low, and C. B. Roth, "Direct Measurement of the Relation between Interlayer Force and Interlayer Distance in the Swelling of Montmorillonite," J. Colloid Interf. Sci., 96, 229 (1983).

Zhang, F., P. F. Low, and C. B. Roth, "Effects of Monovalent Exchangeable Cations and Electrolytes on the Relation between Swelling Pressure and the Interlayer Distance in Montmorillonite," J. Colloid Interf. Sci., 173, 34 (1995).

Zhou, Z., W. D. Gunter, B. Kadatz, and S. Cameron, "Effect of Clay Swelling on Reservoir Quality," Proc. Meeting Petroleum Soc. of CIM, Calgary, Alta., Canada (1994).

Manuscript received June 21, 1996, and revision received Sept. 20, 1996. 